\title{
Probe-Configuration-Dependent Decoherence in an Aharonov-Bohm Ring
}

\author{
Kensuke Kobayashi, Hisashi Aikawa, Shingo Katsumoto ${ }^{\dagger}$, and Yasuhiro Iye ${ }^{\dagger}$ \\ Institute for Solid State Physics, University of Tokyo, 5-1-5 Kashiwanoha, Chiba 27r-8581, Japan \\ ${ }^{\dagger}$ Also at CREST, Japan Science and Technology Corporation, Mejiro, Tokyo 171-0031, Japan
}

(Dated: November 11, 2018)

\begin{abstract}
We have measured transport through mesoscopic Aharonov-Bohm (AB) rings with two different four-terminal configurations. While the amplitude and the phase of the AB oscillations are well explained within the framework of the Landaur-Büttiker formalism, it is found that the probe configuration strongly affects the coherence time of the electrons, i.e., the decoherence is much reduced in the configuration of so-called nonlocal resistance. This result should provide an important clue in clarifying the mechanism of quantum decoherence in solids.
\end{abstract}

PACS numbers: 73.23.Ad,73.63.Nm,85.35.Ds

In mesoscopic physics, it is well known that probes for transport measurements greatly affect the electronic states of the samples. Particularly in single-electron phenomena, a low-impedance probe introduces coupling of the system to an electromagnetic environment and causes the degradation or even disappearance of the singleelectron charging effect [1]. In coherent transport, where probes and samples are strongly coupled, it was also recognized in early studies that "sample" and "leads" are hardly distinguishable [2].

In the coherent regime, however, unlike the case of single-electron phenomena, the quantum coherence of electrons has been believed to be independent of the probe configuration, that is, the selection of the pairs of voltage and current probes in a four-terminal measurement. This assumption is likely to be true for the diffusive transport regime because the electrons experience many elastic scatterings in traversing the sample from one probe to another, which would work as buffers to the environment. Actually, it has been verified both theoretically and experimentally that the phase coherence time $\left(\tau_{\phi}\right)$ in this regime is the same regardless of their probe configurations [3]. On the other hand, in ballistic transport, electrons go into samples directly from the probes, making the boundaries more obscure. In such circumstances, the probes would affect the quantum coherence in the sample by "observation" of electrons through the probes.

Quantum decoherence in solids is now one of the most important issues in physics. Mohanty et al. examined the temperature dependence of $\tau_{\phi}$ in various references and pointed out that saturation of $\tau_{\phi}$ at the lowest temperatures is observed in all cases [4. Subsequent studies have revealed that this saturation is dependent not only on the materials [5] but also on the sample geometry [6], even in the diffusive regime. These suggest a possibility that the saturation is due to some extrinsic effects.

In this Letter, we show that in the ballistic regime the coherence of electrons greatly depends on the probe configuration, through a four-terminal measurement of the same sample with two different probe configurations.

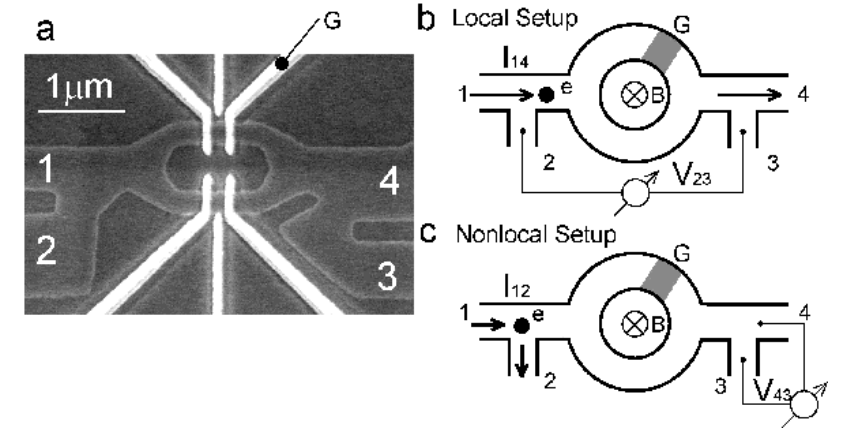

FIG. 1: (a) Scanning electron micrograph of the AB ring. One specific Au/Ti metallic gate marked "G" was used in this study. (b) Local four-terminal setup (current: $1 \rightarrow 4$, voltage: $2 \rightarrow 3$ ). (c) Nonlocal four-terminal setup (current: $1 \rightarrow 2$, voltage: $4 \rightarrow 3)$.

First, we show that the Landauer-Büttiker (LB) formalism, which was constructed for coherent transport with all the probes treated on an equal footing [7], successfully describes the qualitative features of the present experiment. Next, this analysis is shown to lead to an inevitable conclusion that the decoherence is dependent on the probe configuration.

We prepared an AB ring shown in Fig. 11 (a) in a twodimensional electron gas (2DEG) at an AlGaAs/GaAs heterostructure (mobility of $9 \times 10^{5} \mathrm{~cm}^{2} / \mathrm{Vs}$ and sheet carrier density of $3.8 \times 10^{11} \mathrm{~cm}^{-2}$ ) by electron-beam lithography and wet etching. The Fermi wavelength $2 \pi / k_{\mathrm{F}}$ is estimated to be $40 \mathrm{~nm}$. The electron mean free path $l_{e} \sim 8 \mu \mathrm{m}$ is longer than the arms of the ring $L \sim 2 \mu \mathrm{m}$, ensuring that the motion of electrons in the ring is quasi-ballistic. The Au/Ti metallic gates with the width of $W \sim 100 \mathrm{~nm}$ were deposited on the ring to control the $A B$ phase and one specific gate marked " $G$ " in Fig. 11 (a) was used, with the others being kept open in the present study.

In $\mathrm{AB}$ oscillation measurements, electric current is usually passed across the ring and the voltage drop between probes positioned along the current direction is mea- 


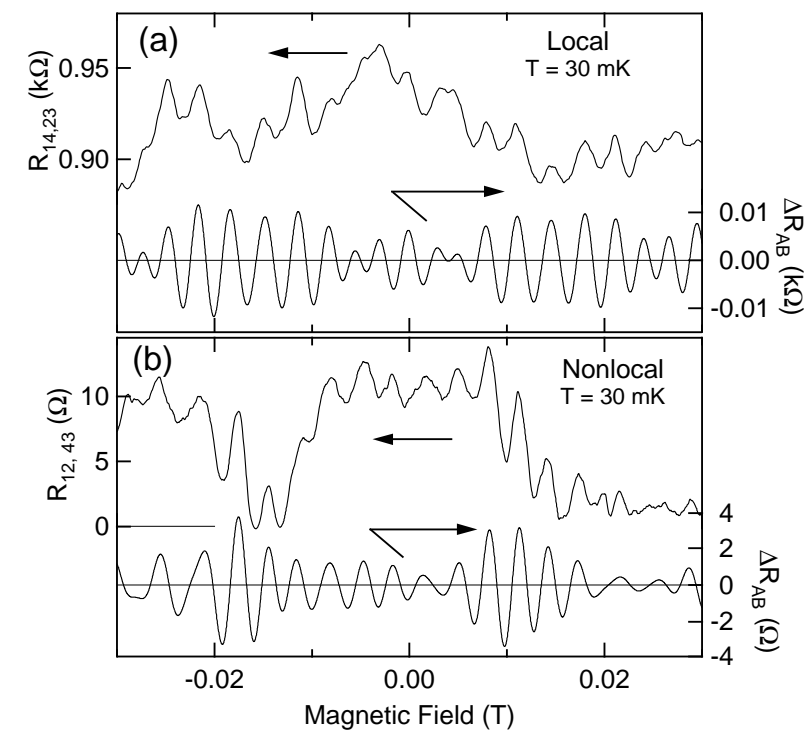

FIG. 2: (a) The upper curve is a typical trace of $R_{14,23}$ in the local setup. The lower curve shows the $\mathrm{AB}$ component $\Delta R_{\mathrm{AB}}$ extracted from $R_{14,23}$ through FFT filtering. (b) Corresponding data for the nonlocal setup.

sured, as illustrated in Fig. 1 (b). Henceforth, we use the notation $R_{i j, k l}$ for the "resistance" measured with the probe pair $(i, j)$ as current probes and the pair $(k, l)$ as voltage probes. Thus, the resistance measured in the configuration in Fig. 1 (b) is denoted as $R_{14,23}$ and is referred to as the "local" setup. Another probe configuration possible for the same sample is the so-called transferresistance configuration shown in Fig. 1 (c), where the current flow is localized at one end of the ring and the voltage appearing in the probe pair at the opposite end is measured ( $R_{12,43}$ and "nonlocal" setup).

In order to minimize the difference due to the instrumental effect, the same setup and parameters were adopted for both configurations except the current amplitudes, which were $3 \mathrm{nA}$ and $15 \mathrm{nA}$ for the local and nonlocal setups, respectively. It was confirmed that these currents do not cause a Joule heating effect. The resistances were measured by the standard lock-in technique. A cryogenic low-pass filter was inserted into every lead at the $1 \mathrm{~K}$ stage. Two samples (\#1 and \#2) with almost the same geometry were measured, yielding essentially the same results.

The curves shown in the upper parts of Figs. 2 (a) and (b) are the raw magnetoresistance traces for the local and nonlocal setups, respectively. The $\mathrm{AB}$ resistance oscillations with a period $\Delta B_{\mathrm{AB}}=3.1 \pm 0.5 \mathrm{mT}$, consistent with the ring size, are superposed on the slower aperiodic fluctuation which arises from small conductance loops within branches. The lower parts of Figs. 目 (a) and (b) present the $\mathrm{AB}$ component $\left(\Delta R_{\mathrm{AB}}\right)$ extracted through fast Fourier transform (FFT). In the local setup, $\Delta R_{\mathrm{AB}}$

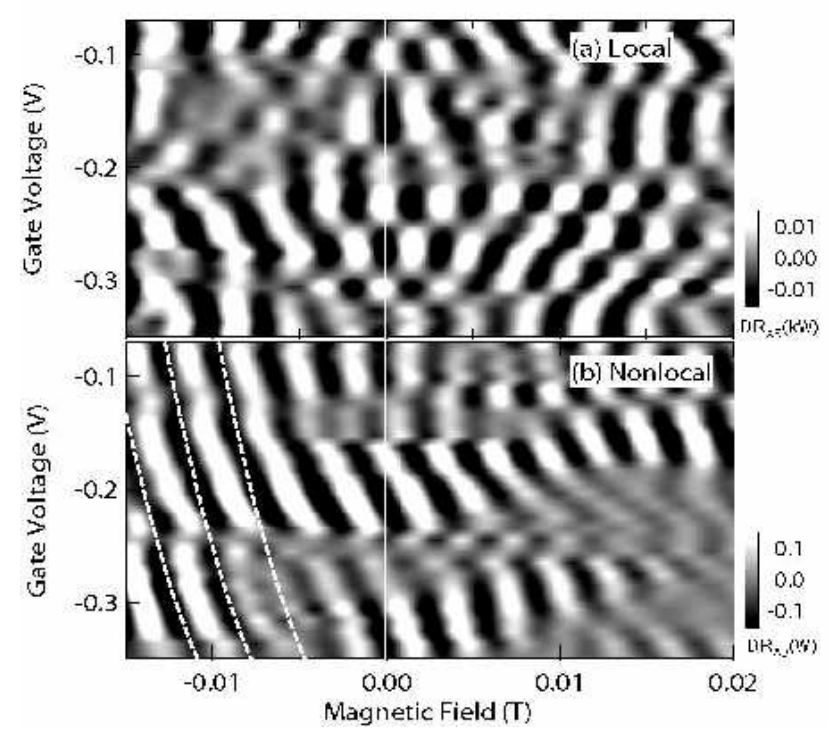

FIG. 3: Gray-scale plots of the $\mathrm{AB}$ oscillation components of the resistances as a function of $V_{\mathrm{G}}$ observed in (a) the local setup and (b) the nonlocal setup. In (a), the $\mathrm{AB}$ oscillation is almost symmetric with respect to $B=0$ (white vertical line). The white dashed curves in (b) are expected equiphase lines in the $B-V_{\mathrm{G}}$ plane.

is only approximately $2 \%$ of the total resistance, which is a typical value for the oscillation amplitude seen in many previous $\mathrm{AB}$ experiments. By contrast, in the nonlocal setup, it averages up to $20 \%$ with a maximum of $\sim 75 \%$. This is the largest $\mathrm{AB}$ amplitude ever reported.

We also performed electrostatic control of the electronic phase through the voltage of the gate $\mathrm{G}\left(V_{\mathrm{G}}\right)$. The gray-scale plots in Fig. 3 show the gate-voltage-induced variation of the $\mathrm{AB}$ oscillation at $30 \mathrm{mK}$. The variation of the electrostatic potential energy by $V_{\mathrm{G}}$ results in that of the kinetic energy, and hence the wave number of the electrons which traverse the region underneath the gate electrode.

Now we demonstrate that the qualitative features of the above results at lowest temperatures are well explained within the LB framework. The LB formula gives $R_{m n, k l}=\left(h / 2 e^{2}\right)\left(T_{k m} T_{l n}-T_{k n} T_{l m}\right) / D$, where $T_{i j}(\geq 0)$ is the transmission coefficient from terminal $j$ to $i$ and the denominator $D$ is a quantity including all of the $T_{i j}$ 's [7]. Here, we note that this formula, which was originally applied to perfect coherent transport [7], can be generalized to include incoherent transport [8]. The generalized LB formula is expressed by the transmission coefficients that are the sum of a coherent part and an incoherent part, and has the same functional form as the original one. Thus the effect of decoherence is appropriately taken into account through the amplitude of the interference term in the transmission coefficients in the LB formula.

From the sample geometry shown in Fig. 1 (a), it is 
natural to make the approximation that $T_{12}=T_{21}=$ $T_{34}=T_{43} \equiv T_{0}, T_{14}=T_{41}=T_{23}=T_{32} \equiv T_{1}$ and $T_{13}=T_{31}=T_{24}=T_{42} \equiv T_{2}$. In order to take the decoherence into account, transmission coefficients for those paths traversing the ring ( $T_{1}$ and $T_{2}$ in the present case) are taken as $T_{i j}=\alpha_{i j}+\beta_{i j} \cos \left(2 \pi \phi / \phi_{0}+\delta_{i j}\right)$, where $\beta_{i j}$ represents the $\mathrm{AB}$ amplitude, $\alpha_{i j}$ the other part of the transmission, $\phi$ the magnetic flux which threads the ring, and $\phi_{0} \equiv h / e$ the flux quantum. $\delta_{i j}$ is the phase due to the difference between the paths. Although $\alpha_{i j}$ also depends on the magnetic field and contains the terms due to the interference, $\left|\overline{\beta_{i j}} / \overline{\alpha_{i j}}\right|$ serves as a good measure of the coherence of traversing electrons, where the bars denote the average over a certain range of magnetic field.

In the above approximation, the LB formula gives the resistances

$$
\begin{aligned}
& R_{14,23}=\frac{h}{2 e^{2}} \frac{T_{0}-T_{2}}{\left(T_{0}+T_{1}\right)\left(T_{1}+T_{2}\right)} \sim \frac{h}{4 e^{2}} \frac{1}{T_{1}+T_{2}}, \\
& R_{12,43}=\frac{h}{2 e^{2}} \frac{T_{1}-T_{2}}{\left(T_{0}+T_{1}\right)\left(T_{0}+T_{2}\right)} \sim \frac{h}{4 e^{2}} \frac{T_{1}-T_{2}}{T_{0}^{2}},
\end{aligned}
$$

where we further approximate that $T_{0} \gg T_{1}, T_{2}$. Note that the information on the phase of the $\mathrm{AB}$ oscillation has been neglected in this approximation because of the symmetric assumption $T_{i j}=T_{j i}$ [8]. Equation (1] indicates that the ratio of the $\mathrm{AB}$ oscillation in $R_{14,23}$ directly reflects $\bar{\beta} / \bar{\alpha}$ while in Eqn. (2) the incoherent parts in $T_{1}$ and $T_{2}$ cancel each other by subtraction and the $\mathrm{AB}$ amplitude is emphasized in the magnetoresistance. This is the qualitative explanation for the results shown in Fig. 2.

Next, the phase modulation by the gate voltage $V_{\mathrm{G}}$ in Fig. 3 is explained as follows. The applied gate voltage modulates the potential of electrons and hence their wave numbers, which results in the variation of $\delta_{i j}$. According to a capacitance model between the gate and the 2DEG [9], the equi-phase curve of the $\mathrm{AB}$ oscillation in the $B-V_{\mathrm{G}}$ plane should take the form $B=$ $\Delta B_{A B}\left(W k_{\mathrm{F}} / 2 \pi\left(1-\left|V_{\mathrm{G}} / V_{C}\right|\right)^{1 / 2}+N\right)(N=0, \pm 1, \ldots)$, where $V_{C}$ is the pinch-off voltage $(-0.435 \mathrm{~V}$ for the present case). This curve satisfactorily explains the overall trend of the phase shift in the nonlocal case as indicated by the white dashed lines in Fig. 3 (b) (the curves for $N=-1,0$, and 1 are plotted). The phase jumps observed in a few regions are due to the intermixing effect of the conduction channels [10].

On the other hand, the phase variation in the local case differs greatly from the above. It was established by early studies that in the LB framework a two-terminal resistance $R_{t t}$ must be an even function of the magnetic field, i.e., $R_{t t}(B)=R_{t t}(-B)$ due to Onsager's reciprocal theorem $T_{i j}(B)=T_{j i}(-B)$ and current conservation [8]. The reciprocal theorem appears in four terminal resistances as $R_{k l, m n}(B)=R_{m n, k l}(-B)$ and $R_{k l, m n}$ itself is not necessarily even with $B$. Hence, it is natural
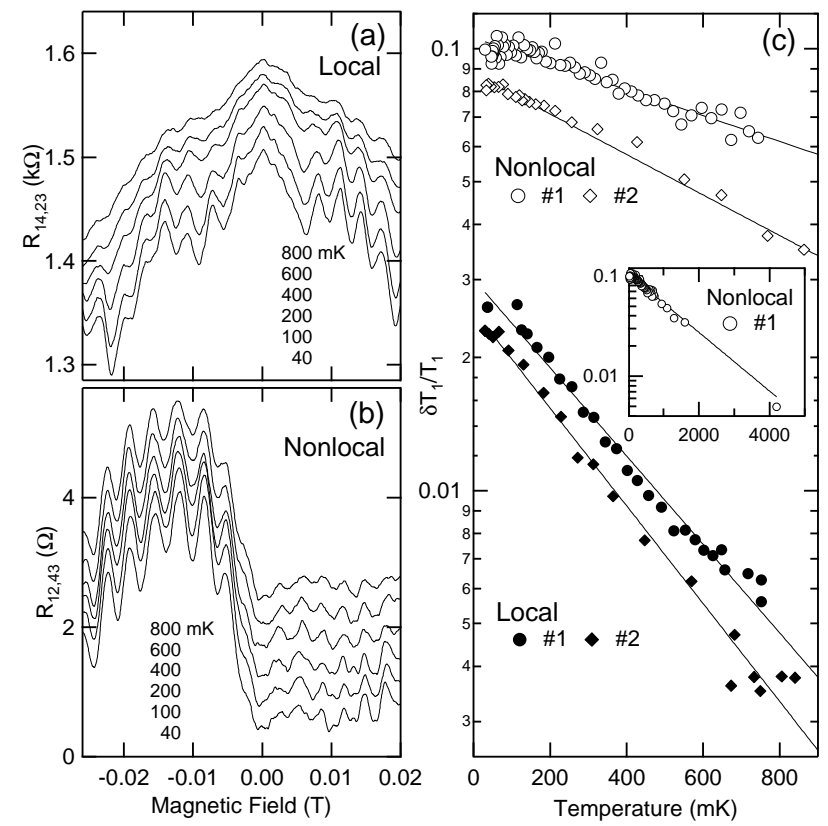

FIG. 4: (a) AB oscillation for the local setup measured at various temperatures. (b) Counterpart of (a) for the nonlocal measurement. The data at $T \geq 100 \mathrm{mK}$ are incrementally shifted upward by $0.02 \mathrm{k} \Omega$ in (a) and $0.4 \Omega$ in (b). The temperature dependence of the $\mathrm{AB}$ effect is clearly different between (a) and (b). (c) Temperature dependence of the portion of the $\mathrm{AB}$ amplitude in the transmission coefficient $\left(\delta T_{1} / T_{1}\right)$ for the two configurations and two different samples (\#1 and \#2) with the same geometry. The solid lines are the results of the fitting to the exponential decay of temperature. The inset shows the nonlocal result up to $4.2 \mathrm{~K}$.

that the phase varies continuously with $V_{\mathrm{G}}$ in the nonlocal setup. Actually, we experimentally confirmed that $R_{12,43}(B)=R_{43,12}(-B)$ holds. In the local setup, the current and voltage probes are placed crossing the ring part, which brings the situation close to the two-terminal case and imposes the symmetry for the magnetic-field reversal. This results in the symmetric pattern with respect to $B=0$ as shown in Fig. 3 (a). As reported previously [10], the phase jump by $\pi$ occurs at several values of $V_{\mathrm{G}}$. Also, the fluctuation of the $\mathrm{AB}$ period due to the mixing of conduction channels causes the quasicontinuous shift of the oscillation though the symmetry for $B=0$ still holds.

Thus far, we have seen that the LB formula successfully describes the qualitative profiles of the results, which leads us to believe that the quantitative outcome of the formula can be used as a basis for the comparison between the two configurations. In order to estimate the classical $T_{i j}$ 's, we measured the resistances for all of the possible probe configurations at $4.2 \mathrm{~K}$ where the quantum interference effect is small. By combining these values with the $\mathrm{AB}$ oscillation measured at various temperatures presented in Figs. 1 (a) and (b), we obtain the 
temperature dependence of the portion of the interference term in the transmission coefficient $\delta T_{1} / T_{1}$ as shown in Fig. 目(c). A striking difference between the local and the nonlocal setup is observed: The ratio of the coherent term to the total transmittance is much higher in the nonlocal probe configuration and it is much more robust against thermal disturbance. As shown in Fig. A (c), the consistency of results between the two different samples with the same geometry strongly indicates that this observation is not sample-specific but is due to the measurement configuration.

Two factors are important for the reduction of the $\mathrm{AB}$ amplitude with temperature. One is the thermal broadening of the electron wave packets, which is expressed in the ballistic case as $\beta_{i j} \propto \exp \left(-\tau_{L} k_{\mathrm{B}} T / \hbar\right) \equiv$ $\exp \left(-\tau_{L} / \tau_{\mathrm{th}}\right)$, where $\tau_{L}=L / v_{\mathrm{F}}\left(v_{\mathrm{F}}\right.$ : the Fermi velocity). The other is the quantum decoherence, which contributes as $\beta_{i j} \propto \exp \left(-\tau_{L} / \tau_{\phi}(T)\right)$ [1]. As shown by the solid lines in Fig. 目 (c), the present data indicate $\beta \propto \exp (-a T)$, implying that $\tau_{\phi}$ is negligible or is also proportional to $T^{-1}$. The obtained values of $a$ are 0.72 and $1.0 \mathrm{~K}^{-1}$ for the nonlocal results, while $a=2.3$ and $2.5 \mathrm{~K}^{-1}$ for the local ones. The thermal broadening affects as $\exp \left(-\tau_{L} / \tau_{\text {th }}\right) \equiv \exp (-b T)$ with $b \sim 1 \mathrm{~K}^{-1}$ in the present case, which would be sufficient to explain the temperature dependence of the nonlocal result. Since the thermal broadening is an intrinsic effect, we attribute the observed probe-configuration-dependent effect to the difference in $\tau_{\phi}$.

Then the next problem is the origin of the difference in $\tau_{\phi}$ between the two setups, or rather the suppression of $\tau_{\phi}$ in the nonlocal setup. Here, we would like to note that the electric current flows differently in that electrons run quasi-ballistically between terminals 1 and 4 in the local setup, while no net current enters the interferometer in the nonlocal setup. In the LB method, zero net current is the consequence of the cancellation of back and forth currents through a sample, but these "currents" are merely means for the calculation and are not physical entities. What actually happens and what the LB formula actually describes in the nonlocal setup is that an electrostatic charge up occurs between electrodes 3 and 4, which prevents the current flow.

We list here two candidates for the origin. The first is that the electrons in the local setup suffer extra decoherence due to the charge fluctuation. It is proposed that in a ballistic $\mathrm{AB}$ ring the charge fluctuations caused by a nearby capacitor such as metallic gates result in decoherence expressed as $\tau_{\phi} \propto T^{-1}$ 11, which is in agreement with our observation. A similar observation was reported by Hansen et al. [12]. In the local setup, charge fluctuation, which originates from shot noise [13] due to the nonequilibrium current in the interferometer, is possible to play a significant role when the net current exists. In the nonlocal setup, this mechanism is expected to be suppressed. The second mechanism is based on the deduc- tion that in the local setup injected electrons traverse the ring quasi-ballistically and may retain some traces that can tell "which path they went through" to the circumambient electrons in 2DEG 114. In other words, the interference does not complete at the outlet of the sample locally. This should lead to a loss of coherence through the process discussed by Stern et al. 15]. In the nonlocal setup, the electrons in the ring are comparatively isolated from the leads by the electrostatic barrier formed by the charge up. This mechanism may be reduced by making the outlet narrower to prevent the leakage of the "which path" information.

In conclusion, we have observed that the quantum decoherence in a ballistic $\mathrm{AB}$ ring is dependent on the probe configuration. While $\tau_{\phi}$ behaves as $\propto T^{-1}$, in both local and nonlocal setups, the coherence survives at much higher temperatures in the nonlocal setup. We believe that the key difference is the amplitude of the net electric current, and plausible mechanisms are discussed. While theoretical effort is necessary to clarify this issue, the LB formula successfully describes the qualitative profiles of the results, such as the large amplitude of the $\mathrm{AB}$ oscillation in the nonlocal setup or the phase tuning by electrostatic potential.

We thank H. Ebisawa and H. Fukuyama for helpful discussion. This work is partly supported by a Grantin-Aid for Scientific Research and by a Grant-in-Aid for COE Research ("Quantum Dot and Its Application") from the Ministry of Education, Culture, Sports, Science, and Technology.

[1] M. Watanabe and D. B. Haviland, Phys. Rev. Lett. 86, 5120 (2001).

[2] C. P. Umbach, P. Santhanam, C. van Haesendonck, and R. A. Webb, Appl. Phys. Lett. 50, 1289 (1987).

[3] H. Haucke et al., Phys. Rev. B 41, 12454 (1990) and references therein.

[4] P. Mohanty et al., Phys. Rev. Lett. 78, 3366 (1997).

[5] A. B. Gougam et al., J. Low Temp. Phys. 118, 447 (2000).

[6] D. Natelson, R. L. Willett, K. W. West, and L. N. Pfeiffer, Phys. Rev. Lett. 86, 1821 (2001).

[7] M. Büttiker, Phys. Rev. Lett. 57, 1761 (1986).

[8] M. Büttiker, IBM J. Res. Develop. 32, 317 (1988).

[9] A. Yacoby, U. Sivan, C. P. Umbach, and J. M. Hong, Phys. Rev. Lett. 66, 1938 (1991).

[10] G. Cernicchiaro et al., Phys. Rev. Lett. 79, 273 (1997).

[11] G. Seelig and M. Büttiker, Phys. Rev. B 64, 245313 (2001).

[12] A. E. Hansen et al., Phys. Rev. B 64, 45327 (2001).

[13] M. Reznikov et al., Superlattices Microstruct. 23, 901 (1998).

[14] E. Buks et al., Nature 391, 871 (1998).

[15] A. Stern, Y. Aharonov, and Y. Imry, Phys. Rev. A 41, 3436 (1990). 